\title{
Excitation Function of Energy Density and Partonic Degrees of Freedom in Relativistic Heavy Ion Collisions
}

\author{
H. Weber, C. Ernst, M. Bleicher*, L. Bravina ${ }^{\dagger}$, H. Stöcker and W. Greiner \\ Institut für Theoretische Physik \\ Johann Wolfgang Goethe Universität \\ Robert Mayer Str. 8-10 \\ D-60054 Frankfurt am Main, Germany \\ C. Spieles ${ }^{\ddagger}$ \\ Lawrence Berkeley Laboratory \\ 1 Cyclotron Road \\ Berkeley, CA 94720, USA \\ S.A. Bass ${ }^{\ddagger}$ \\ Department of Physics \\ Duke University \\ Durham, NC, 27708-0305, USA
}

\begin{abstract}
We estimate the energy density $\epsilon$ pile-up at mid-rapidity in central $\mathrm{Pb}+\mathrm{Pb}$ collisions from $2-200 \mathrm{GeV} /$ nucleon. $\epsilon$ is decomposed into hadronic and partonic contributions. A detailed analysis of the collision dynamics in the framework of a microscopic transport model shows the importance of partonic degrees of freedom and rescattering of leading (di)quarks in the early phase of the reaction for $E_{l a b} \geq 30 \mathrm{GeV} /$ nucleon. In $\mathrm{Pb}+\mathrm{Pb}$ collisions at 160 $\mathrm{GeV} /$ nucleon the energy density reaches up to $4 \mathrm{GeV} / \mathrm{fm}^{3}, 95 \%$ of which are contained in partonic degrees of freedom.
\end{abstract}

*Fellow of the Josef Buchmann Foundation

$\dagger$ Fellow of the Alexander v. Humboldt Foundation

${ }^{\ddagger}$ Feodor Lynen Fellow of the Alexander v. Humboldt Foundation 
The determination of energy densities in ultra-relativistic heavy-ion collisions is crucial for any discussion involving a possible deconfinement phase transition to a QGP [1] [7]. Energy densities are not directly measurable - therefore a comparison between data and models must be used to pin down the time evolution of the reaction. Then, estimates for the energy density during the hot and dense early reaction stage [9,8, 4, 6] can be obtained from the most reasonably looking models.

It has been questioned whether hadronic transport models are still valid at CERN/SPS energies: the energy density estimates obtained in these frameworks are claimed to be well above the critical energy density estimates for a deconfinement phase transition given by Lattice Gauge Theory 10 12. Hadronic transport models, however, contain implicit partonic degrees of freedom [13] - particle production at high energies is e.g. modeled via the production and fragmentation of strings [14 [16]. This issue is studied in the present paper.

In the UrQMD model used below, the leading hadrons of the fragmenting string contain the valence-quarks of the original excited hadron. These leading hadrons are allowed - in the model - to interact even during their formation time, with a reduced cross section, thus accounting for the original valence quarks contained in that hadron. Those leading hadrons represent a simplified picture of the leading (di)quarks of the fragmenting string. Newly to-be-produced hadrons which do not contain string valence quarks do in the present model not interact during their formation time - however, they contribute to the energy density of the system. A proper treatment of the partonic degrees of freedom during the formation time ought to include soft and hard parton scattering [28 and the explicit time-dependence of the color interaction between the expanding quantum wave-packets [24]: However, such an improved treatment of the internal hadron dynamics has not been implemented for light quarks into the present model. Therefore, in the following analysis all contributions stemming from hadrons within their formation time are termed "partonic". All contributions stemming from fully formed hadrons are termed "hadronic". The main focus of this paper is on the partitioning and the time evolution of the energy density and the collision dynamics of the early, intermediate, and late reaction stage at energies $E_{l a b}=10-200 \mathrm{GeV} /$ nucleon.

For our analysis we employ the UrQMD model [17], which is based on analogous principles as (Relativistic) Quantum Molecular Dynamics [18 23. Hadrons are represented by Gaussians in phase space and are propagated according to Hamilton's equation of motion. The collision term of the UrQMD model treats 55 different isospin ( $\mathrm{T}$ ) degenerate baryon (B) species (including nucleon-, delta- and hyperon- resonances with masses up to $2 \mathrm{GeV}$ ) and 32 different T-degenerate meson (M) species, including (strange) resonances as well as their corresponding anti-particles, i.e. full baryon-antibaryon symmetry is included. Isospin is treated explicitly. For hadronic excitations with masses $m>2 \mathrm{GeV}(\mathrm{B})$ and $>1.5 \mathrm{GeV}$ (M) a string model is used. Particles produced in the string fragmentation are assigned a formation time. This time $\tau_{f}$ physically consists of a quantal time $\tau_{Q}$, i.e. before the partons are produced, $\tau_{Q} \sim 1 / m$, and a quantum diffusion time, $\tau_{D}$, during which the partons evolve in the medium to build up their internal asymptotic wave-functions to form the hadron. $\tau_{Q}$ and $\tau_{D}$ differ for different parton and hadron species. For our present purpose, we - for the sake of simplicity - just collect all partons, formed and unformed, as one species. For a detailed overview of the elementary cross sections and string excitation scheme included in the UrQMD model, see ref. [17]. 
The partitioning of the distinct constituents can be inspected in figure 1 which shows the time-evolution of the energy density for central $\mathrm{Pb}+\mathrm{Pb}$ collisions at $160 \mathrm{GeV} /$ nucleon. The nuclei are initialized such that they touch a $t=0 \mathrm{fm} / \mathrm{c}$. The energy density is partitioned into the above defined "hadronic" contribution, from fully formed hadrons, and the "partonic" contribution, from partons, constituent quarks and diquarks within the hadron formation time. Nearly all incident baryons are rapidly excited into strings. Subsequently, "partonic" energy density builds up, reaching values of $4 \mathrm{GeV} / \mathrm{fm}^{3}$ around midrapidity, $\Delta y=1$ (at $t \approx 1 \mathrm{fm} / \mathrm{c}$ ). In the course of the reaction hadrons are formed which increases in the "hadronic" energy density, accompanied by a nearly exponential decrease in the "partonic" energy density.

These energy densities are calculated as follows: In the UrQMD model hadrons are represented by Gaussian wave packets. The width of the Gaussians $\sigma=1.04 \mathrm{fm}$ and their normalization are chosen such that a calculation of the baryon density in the initial nuclei yields ground state nuclear matter density. The (energy-) densities in the central reaction zone are obtained by summing analytically over all Gaussian hadrons around mid-rapidity $\left(y_{c . m .} \pm 1\right)$ at the locations of these hadrons and then averaging over these energy densities. This summation over Gaussians yields a smooth estimate for baryon- and energy-densities, as compared to counting hadrons in a test volume. The rapidity cut insures that only those particles are taken into account which have interacted. Thus, the free streaming "spectator" matter is discarded.

The absolute value of the energy density, however, may depend on the rapidity cut: Without rapidity cut the energy densities during the early reaction stage $(t \approx 1 \mathrm{fm} / \mathrm{c})$ can be as high as $20 \mathrm{GeV} / \mathrm{fm}^{3}$. Even higher values in $\epsilon$ can be obtained by choosing the geometric center of the collision for the sum over the Gaussians instead of averaging over the energy densities at the locations of the hadrons. The energy density at a single point may not be physically meaningful and therefore the latter method is favorable.

Calculating the energy density by summing over particles in the central reaction cell may yield values for the total energy density of up to $30 \mathrm{GeV} / \mathrm{fm}^{3}$ (for a cell of $2 \times 2 \times 1 \mathrm{fm}^{3}$ ). However, the values depend strongly on the volume of the cell.

The time evolution of partonic constituents and hadrons is shown in the upper frame of figure 2. The first $5 \mathrm{fm} / \mathrm{c}$ of the reaction are dominated by the partonic constituents. The long-dashed and the dotted curves show the number of baryons and mesons contained in those constituents. In the case of leading-particles these can be interpreted as constituent (di)quarks or, for freshly born partons with small cross sections, as excitation modes of the color field.

The lower frame of figure 2 shows the time evolution of the number of baryon-baryon (BB), meson-baryon (MB) and meson-meson (MM) collisions, both for "hadronic" and "partonic" interactions. "Partonic" interactions denote interactions of leading (di)quarks either among themselves or with fully formed hadrons. The early reaction stages, especially the MM and MB cases, are clearly dominated by those "partonic" interactions. First after $\approx 10 \mathrm{fm} / \mathrm{c}$ do collisions among fully formed hadrons dominate the collision dynamics. This number increases further if the scattering of the newly formed partons is included. Thus "partonic" degrees of freedom significantly contribute both, to the energy density, as well as to the collision dynamics in the first $5 \mathrm{fm} / \mathrm{c}$.

It should be noted that the "partonic" collision rates strongly depend on the treatment 
of the partonic cross section during formation time: In this analysis all interactions during formation time have been considered purely "partonic". Other scenarios, however, include a "hadronic" contribution to the cross section which increases continuously during $\tau_{D}$ and reaches its full hadronic value at the end of $\tau_{D}$ [24].

The multiplicities of partons/hadrons actually contained in the region of high energy density are shown in figure 3 for different incident beam energies: We define this region to have a "partonic" energy density of $\epsilon_{Q} \geq 2 \mathrm{GeV} / \mathrm{fm}^{3}$ and plot the multiplicity of "constituent quarks" propagating through this energy density. The number of "constituent quarks" is obtained by summing over all selected partonic constituents, weighting the developing baryons by a factor of 3 and mesons-to-be by a factor of 2 , respectively. This allows to estimate the volume of high "partonic" energy density: at $160 \mathrm{GeV}$, more than 1000 "valence quarks" are present over a time scale of $\Delta t \approx 2 \mathrm{fm} / \mathrm{c}$ at $\epsilon_{Q} \geq 2 \mathrm{GeV} / \mathrm{fm}^{3}$. Already at beam energies around $40 \mathrm{GeV} /$ nucleon, a sizable "partonic" phase exists; at $10.6 \mathrm{GeV} /$ nucleon, however, the number of "constituent quarks" propagating through high "partonic" energy density $\epsilon_{Q} \geq 2 \mathrm{GeV} / \mathrm{fm}^{3}$ is negligible.

However, during the early reaction stages matter in the central reaction volume is neither fully hadronic, nor thermally and chemically equilibrated. A detailed analysis of velocity distributions and chemical composition in a central cell for $\mathrm{Au}+\mathrm{Au}$ reactions at 10.6 $\mathrm{GeV} /$ nucleon 25] shows that first at times $t \approx 10 \mathrm{fm} / \mathrm{c}$ hadronic matter in the central cell can be viewed as nearly chemically equilibrated. This matter however never exceeds energy densities of $\sim 1 \mathrm{GeV} / \mathrm{fm}^{-3}$, i.e. a density above which the notion of separated hadrons loses its meaning.

Do "partonic" degrees of freedom play any role at $10 \mathrm{GeV} /$ nucleon, i.e. at the AGS? The upper frame of figure 1 shows the maximum total energy density obtained in central collisions of heavy nuclei as a function of incident beam energy, starting from $2 \mathrm{GeV} /$ nucleon and going up to $200 \mathrm{GeV} /$ nucleon. The energy density is obtained by the same method as used figure 11. However, here "partonic" and "hadronic" contributions have been summed. $\epsilon$ increases monotonously with the beam energy, reaching values as high as $4 \mathrm{GeV} / \mathrm{fm}^{3}$ for SPS energies, which would seem unreasonably high, if a purely hadronic scenario were used.

The lower frame of figure 4 shows the maximum fraction of the energy density which is contained in "partonic" degrees of freedom. Even at AGS energies, already more than half of the energy density is due to such "partonic" degrees of freedom, even though these do not yet dominate the "hadronic" contributions. At $40 \mathrm{GeV} /$ nucleon, the maximum of the fraction of "partonic" energy density is already $>90 \%$ of the total $\epsilon$.

The monotonous increase of the energy density excitation function does not imply that the excitation function of the space-time volume of high baryon density shows the same behavior. At AGS energies, $E_{l a b} \sim 10 \mathrm{GeV} /$ nucleon, baryons still dominate the composition of the hadronic matter, whereas at CERN/SPS energies, $200 \mathrm{GeV} /$ nucleon, mesons constitute the largest fraction of the hadronic matter. The maximum space-time volume of dense baryonic matter can be reached at beam energies around $40 \mathrm{GeV} /$ nucleon. A detailed analysis of that regime, also with respect to experimental signatures, is presently underway [26].

The importance of "partonic" degrees of freedom for the collision dynamics in ultrarelativistic heavy ion collisions at CERN/SPS energies does not imply that an equilibrated Quark-Gluon-Plasma has been formed. In the UrQMD approach the "partonic" phase has been modeled as an incoherent superposition of non-interacting partonic constituents. 
Furthermore, these "partons" retain their original correlation into hadrons - deconfinement is not implemented into the present UrQMD approach. The leading (di)quark interactions (among each other and with fully formed hadrons) constitute an interacting "mixed phase" (for the constituent parton dynamics in this model, see, however [24,27]). In contrast, parton cascades [28,29] allow for interactions among the partons only, while hadronic final state interactions are to a large extent neglected. Introducing the parton color dynamics, e.g. via an increasing cross section for partonic constituents according to the QCD factorization theorem is a step towards such a more complete scenario [24], including both parton- and hadron rescattering, thus allowing ultimately also for a detailed study of the equilibration of the constituents.

The question to be studied in such a model would be whether the partonic phase in relativistic heavy ion collisions rather resembles more a non-interacting free streaming parton gas or a strongly interacting, nearly equilibrated "soup" of partons, which so far has been the prevalent simplified picture of a QGP.

S.A.B. acknowledges helpful discussions with Berndt Müller. This work has been supported by GSI, BMBF, Graduiertenkolleg "Theoretische und experimentelle Schwerionenphysik" DFG and DOE grant DE-FG02-96ER40945. 


\section{REFERENCES}

[1] J. C. Collins and M. Perry, Phys. Rev. Lett. 34, 1353 (1975).

[2] H. Stöcker, G. Graebner, J.A. Maruhn and W. Greiner, Phys. Lett. B95, 192 (1980).

[3] E. V. Shuryak, Phys. Rep. 61, 71 (1980).

[4] L. McLerran, Rev. Mod. Phys. 58, 1021 (1986).

[5] H. Stöcker and W. Greiner, Phys. Rep. 137, 277 (1986).

[6] R. R. Clare and D. Strottman, Phys. Rep. 141, 179 (1986).

[7] K. Kajantie and L. McLerran, Ann. Rev. Nucl. Part. Sci. 37, 293 (1987).

[8] J. D. Bjorken, Phys. Rev. D27, 140 (1983).

[9] L. van Hove, Phys. Lett. B118, 138 (1982).

[10] T. Blum, L. Kärkkäinen, D. Toussaint, and S. Gottlieb, Phys. Rev. D51, 5153 (1995).

[11] G. Boyd et al., Phys. Rev. Lett. 75, 4169 (1995).

[12] E. Laermann, Nucl. Phys. A610, 1c (1996).

[13] H. Sorge, A. v. Keitz, R. Mattiello, H. Stöcker and W. Greiner, Z. Phys. C47 629 (1990).

[14] B. Andersson, G. Gustavson, and B. Nilsson-Almquist, Nucl. Phys. B281, 289 (1987).

[15] B. Andersson et al., Comp. Phys. Comm. 43, 387 (1987).

[16] T. Sjoestrand, Comp. Phys. Comm. 82, 74 (1994).

[17] S. A. Bass, M. Belkacem, M. Bleicher, M. Brandstetter, L. Bravina, C. Ernst, L. Gerland, M. Hofmann, S. Hofmann, J. Konopka, G. Mao, L. Neise, S. Soff, C. Spieles, H. Weber, L. A. Winckelmann, H. Stöcker, W. Greiner, C. Hartnack, J. Aichelin and N. Amelin, Prog. Part. Nucl. Phys. 41, 225 (1998), nucl-th/9803035.

[18] G. Peilert, A. Rosenhauer, H. Stöcker, W. Greiner, and J. Aichelin, Modern Physics Letters A3, 459 (1988).

[19] C. Hartnack, L. Zhuxia, L. Neise, G. Peilert, A. Rosenhauer, H. Sorge J. Aichelin, H. Stöcker and W. Greiner, Nucl. Phys. A495, 303 (1989).

[20] J. Aichelin, Phys. Rep. 202, 233 (1991).

[21] H. Sorge, H. Stöcker, and W. Greiner, Ann. Phys. 192, 266 (1989).

[22] H. Sorge, Phys. Rev. C52, 3291 (1995), nucl-th/9509007.

[23] E. Lehmann, R. Puri, A. Faessler, G. Batko, and S. Huang, Phys. Rev. C51, 2113 (1995).

[24] L. Gerland, L. Frankfurt, M. Strikman, H. Stöcker, and W. Greiner, Phys. Rev. Lett. 81, 762 (1998).

[25] L.V. Bravina, M.I. Gorenstein, M. Belkacem, S.A. Bass, M. Bleicher, M. Brandstetter, M. Hofmann, S. Soff, C. Spieles, H. Weber, H. Stöcker and W. Greiner. Phys. Lett. B (1998) in print, nucl-th/9804008.

[26] H. Weber, S.A. Bass, M. Bleicher, C. Spieles, H. Stöcker and W. Greiner, manuscript in preparation.

[27] C. Spieles, L. Gerland, N. Hammon, M. Bleicher, S.A. Bass, H. Stöcker, W. Greiner, C. Lourenco and R. Vogt, Euro. Phys. J. C, in print and eprint hep-ph/9706525.

[28] K. Geiger and B. Müller, Nucl. Phys. B369, 600 (1992).

[29] K. Geiger, Comput. Phys. Commun. 104, 70 (1997), hep-ph/9701226. 


\section{FIGURES}

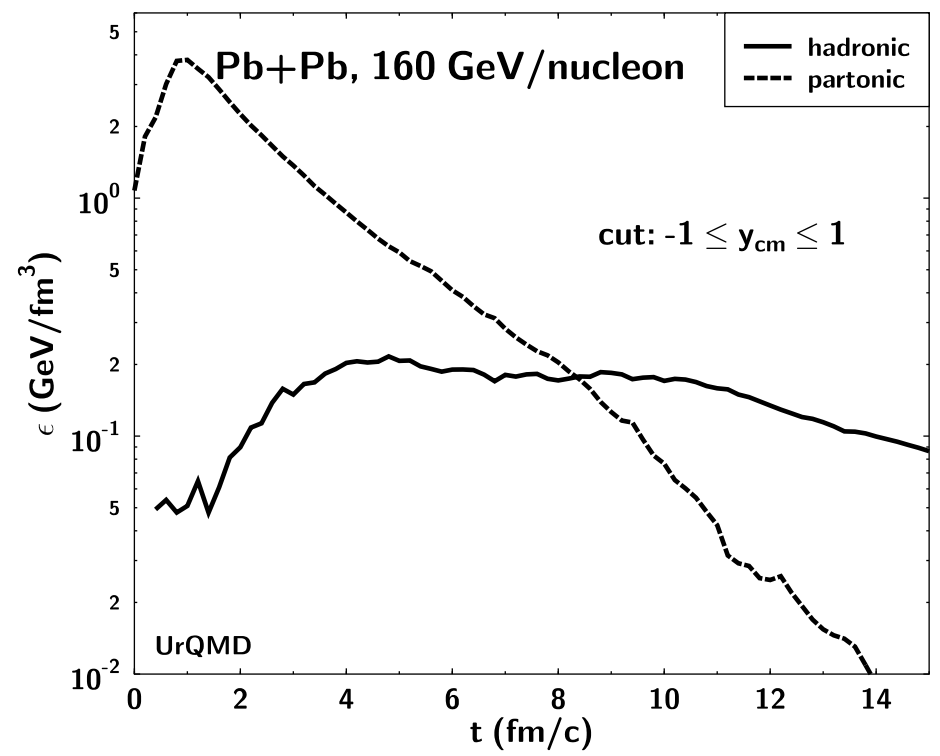

FIG. 1. Time evolution of the energy density $\epsilon$ in central $\mathrm{Pb}+\mathrm{Pb}$ reactions at $160 \mathrm{GeV} /$ nucleon. $\epsilon$ has been decomposed into "partonic" and "hadronic" contributions and only particles around mid-rapidity have been taken into account. The early and intermediate reaction stages are dominated by the "partonic" contribution. 


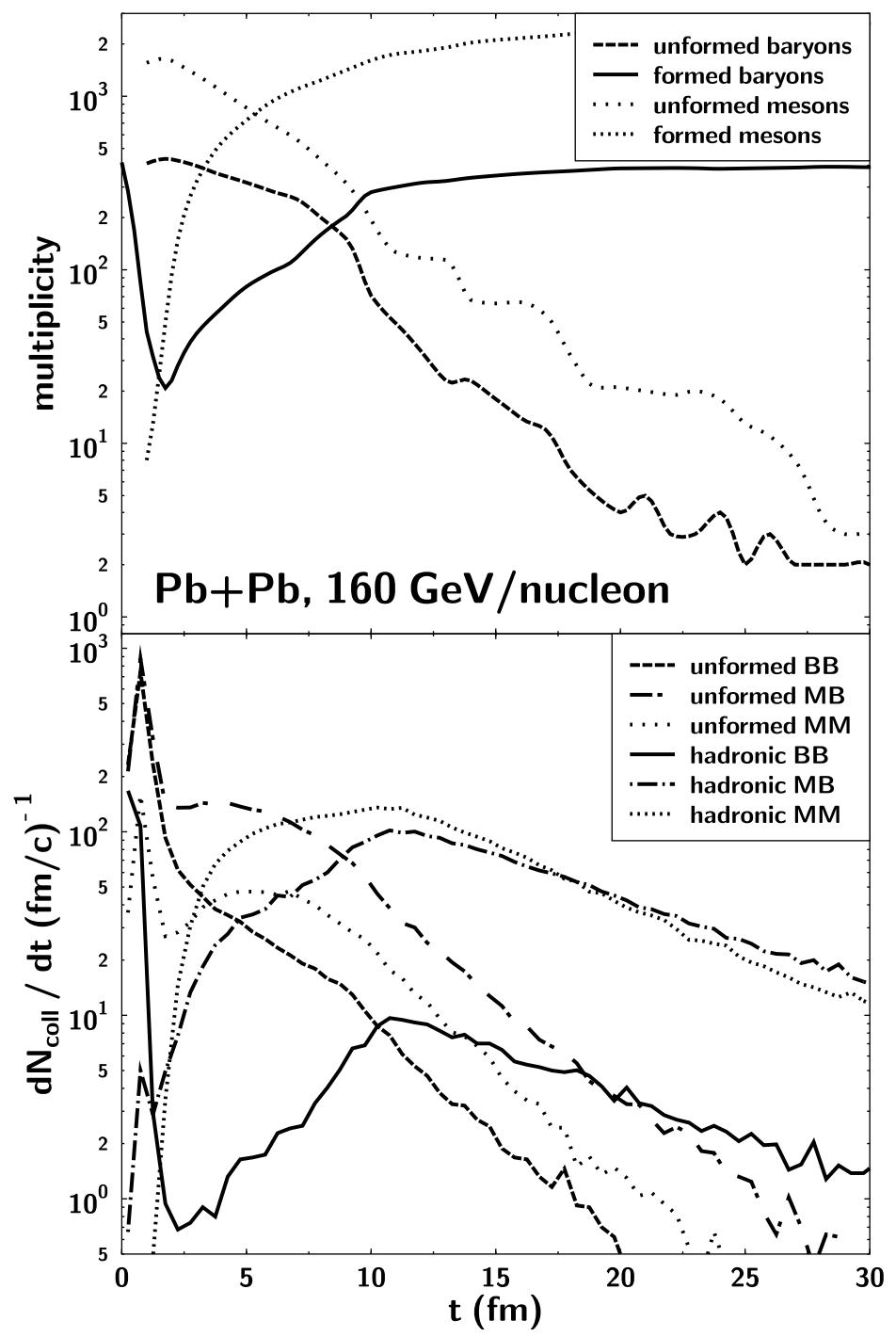

FIG. 2. Top: Time evolution of the multiplicity of hadrons and partonic constituents, divided into baryonic and mesonic contributions. Bottom: Collision rates for baryon-baryon (BB) and meson-meson (MM) collisions. The rates have been decomposed into interactions involving formed hadrons and those involving partonic constituents. 


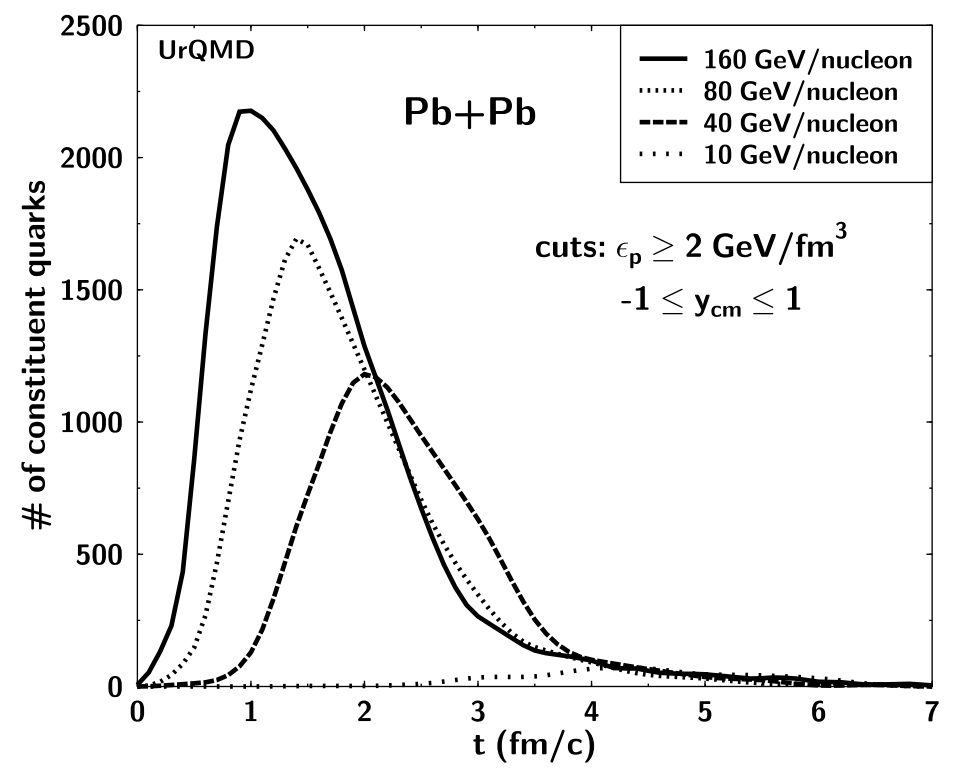

FIG. 3. Multiplicity of "constituent quarks" propagating through a "partonic" energy density of $\epsilon_{Q} \geq 2 \mathrm{GeV} / \mathrm{fm}^{3}$. The number of "constituent quarks" is obtained by summing over all partonic constituents, weighting the developing baryons by a factor of 3 and mesons-to-be by a factor of 2 , respectively. At $160 \mathrm{GeV}>1000$ "constituent quarks" are present over a time scale of $\Delta t \approx 2$ $\mathrm{fm} / \mathrm{c}$. 


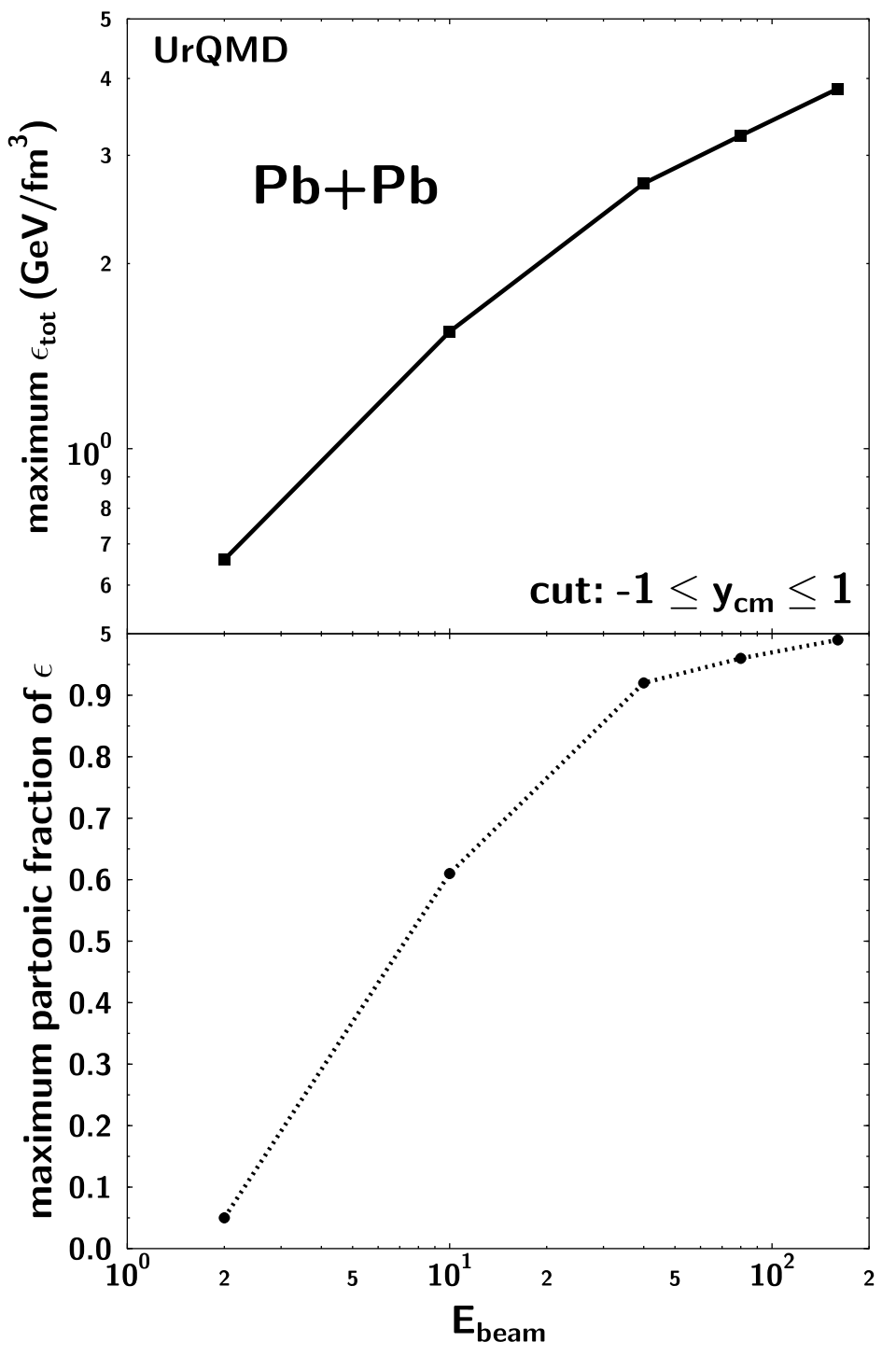

FIG. 4. Top: excitation function of the maximum total energy density mid-rapidity hadrons experience. Bottom: excitation function of the maximum "partonic" fraction of energy density. Already at a beam energy of $40 \mathrm{GeV} /$ nucleon more than $90 \%$ of the energy density is contained in partonic degrees of freedom at one time during the collision. 